Research Article

\title{
To evaluate and compare the hypoglycemic, antioxidant and hypolipidemic effect of lycopene with atorvastatin in hyperlipidemic New Zealand white rabbits
}

\author{
Sarita Mulkalwar*, Bhalchandra Rane, Niranjan Munjal, Pravin Golande, Lopamudra Behera
}

Department of Pharmacology, Dr. D. Y. Patil Medical College, Pimpri, Pune, Maharashtra, India

Received: 21 December 2014 Accepted: 11 January 2015

*Correspondence to:

Dr. Sarita Mulkalwar,

Email: smulkal@hotmail.com

Copyright: (C) the author(s), publisher and licensee Medip Academy. This is an openaccess article distributed under the terms of the Creative Commons Attribution NonCommercial License, which permits unrestricted noncommercial use, distribution, and reproduction in any medium, provided the original work is properly cited.

\begin{abstract}
Background: During recent years, the carotenoid lycopene has attracted much attention for its potentially beneficial cardiovascular effects, which is located mainly in tomato peels. The antioxidant properties of tomatoes has been extensively studied for the prevention of cardiovascular disease, but the beneficial effects of pure lycopene supplement is still debatable. Therefore, this study was planned to evaluate and compare the hypoglycemic, hypolipidemic and antioxidant effects of pure lycopene powder and to compare it with that of atorvastatin in hyperlipidemic New Zealand white rabbits.

Methods: Adult male New Zealand white rabbits $(1.5-2.5 \mathrm{~kg})$ were divided into three groups of six animals each. Group I - High fat diet (HFD) $(5 \mathrm{ml} / \mathrm{kg})$. Group II - HFD $(5 \mathrm{ml} / \mathrm{kg})+$ lycopene $(10 \mathrm{mg} / \mathrm{kg})$ orally. Group III - HFD $(5 \mathrm{ml} / \mathrm{kg})+$ atorvastatin $(5 \mathrm{mg} / \mathrm{kg})$ orally. Blood samples were taken from all 18 rabbits for baseline estimation of blood sugar levels (BSL), lipid levels and blood superoxide dismutase levels. Same tests were performed in all groups after 6 weeks.

Results: The lipid lowering activity of atorvastatin $5 \mathrm{mg} / \mathrm{kg}$ was better than that of lycopene while the antioxidant activity of lycopene was better than that of atorvastatin. Both the results were statistically significant. There was no significant difference in fasting BSLs in both the groups.

Conclusion: Findings suggest that lycopene may have considerable therapeutic benefit as an antioxidant and hypolipidemic agent, but may not be effective as a hypoglycemic agent.
\end{abstract}

Keywords: Antioxidant, Superoxide dismutase, High fat diet

\section{INTRODUCTION}

Despite the changes in lifestyles and the use of new pharmacologic approaches to lower cholesterol levels, cardiovascular disease (CVD) continues to be the principal cause of death. Nearly 17 million deaths are attributed to this disease. CVD is a collective term for all the diseases of heart and blood vessels. ${ }^{1}$ Numerous epidemiological investigations have characterized the risk pattern for coronary heart disease. In particular age, sex, elevated low-density lipoprotein cholesterol (LDL-C) levels, low high-density lipoproteincholesterol (HDL-C) levels, diabetes mellitus and cigarette smoking are the key risk factors for coronary heart disease. ${ }^{2}$

Atherosclerosis is one of the most widespread conditions that threaten human health and survival. The basic pathogenesis involves an insult to the endothelial and smooth muscle cells of the arterial wall by various harmful factors such as viral infection, mechanical damage and dyslipidemia, especially abnormal oxidized LDL levels, leading to an excessive chronic inflammatory or fibro proliferative response. The pathologic process results in a progressive accumulation of lipids and fibrous elements in the large arteries. ${ }^{3}$ Hypercholesterolemia is a known major risk factor for atherosclerosis. It can result from endogenous causes or from exogenous dietary source. Dietary hypercholesterolemia may result from increased intake of cholesterol or saturated fatty acids. Hypercholesterolemia, regardless of cause, influences the development of atherosclerosis. ${ }^{4}$

There is convincing scientific evidence in support of the association between diet and chronic diseases. Based which, dietary guidelines have been formulated around the world for prevention of chronic diseases such as cancer, CVDs, diabetes and osteoporosis. One of their main recommendations of is to increase the consumption of fruits and vegetables that are good sources of carotenoids and other biologically active phytochemicals. In recent years, oxidative 
stress induced by reactive oxygen species (ROS) generated by normal metabolic activity as well as lifestyle factors such as smoking, exercise and diet, has been implicated in the causation and progression of several chronic diseases. Antioxidants that can mitigate the damaging effects of ROS have been the focus of research. ${ }^{5}$ Carotenoids are a family of pigmented compounds synthesized by plants and microorganisms, but not by animals. Fruits and vegetables constitute the major source of carotenoids in the human diet. They are present as micro components in fruits and vegetables and are responsible for their colors. ${ }^{6}$

More than 600 carotenoids have so far been identified in nature. However, only about 40 are present in typical human diet, of which about 20 have been identified in the human blood and tissues. Close to $90 \%$ of these carotenoids in the diet and the human body are represented by $\beta$-carotene, $\alpha$-carotene, lycopene, lutein and cryptoxanthin. ${ }^{7}$

Lycopene is an unsaturated acyclic carotenoid with 11 linear conjugated and 02 non-conjugated double bonds. It is the pigment that gives red color to fruits and vegetables such as tomatoes, watermelons, grapefruits and red grapes. It is reported to be the most efficient single oxygen quencher in carotenoids group, whose quenching ability is mainly dependent on the number of conjugated double bonds, and to a lesser extent on the presence of cyclic or acyclic end groups. ${ }^{8}$

3-hydroxy-3-methylglutaryl coenzyme A (HMG-CoA) reductase inhibitors, such as atorvastatin are highly effective lipid-lowering drugs. Large scale controlled clinical studies have established that LDL-C lowering with HMG-CoA reductase inhibitors has a beneficial impact on primary and secondary prevention of coronary artery disease (CAD), reducing $\mathrm{CAD}$ risk, disease progression, myocardial events and mortality. Atorvastatin is the most efficacious of the currently available HMG-CoA reductase inhibitors in terms of its LDL-C lowering effect. In addition, it has a modest triglyceride (TG) lowering action. ${ }^{9}$

Although the antioxidant properties of tomatoes and processed tomato products have been extensively studied for the prevention of CVD, the beneficial effects of pure lycopene supplement are still debatable. Despite the spotlight on lycopene, it may not be protective on its own. It may only be a marker for other active substances in tomatoes, or it may work with other phytonutrients to confer health benefits. Hence, the present study aims to evaluate the hypolipidemic, hypoglycemic and antioxidant effect of pure lycopene powder in hyperlipidemic rabbits and to compare the same with that of atorvastatin.

\section{METHODS}

Male New Zealand white rabbits $(n=18)$ and weighing $1.5-2.5 \mathrm{~kg}$ were used. Rabbits were housed individually in standard stainless steel cages at $24^{\circ} \mathrm{C}$ with a $12 \mathrm{hrs}$ light: dark cycle and had free access to food and tap water. Studies were carried out as per guidelines by Committee for the Purpose of Control and Supervision of Experiments on Animals, New Delhi (India). The study was approved by Institutional Animal Ethics Committee, Dr. D.Y Patil Medical College, Pimpri, Pune. All rabbits were allowed to acclimatize for 1 week after which blood samples were collected for baseline estimation of blood sugar level (BSL), lipids (total cholesterol [TC], TG, LDL and HDL) and antioxidant (superoxide dismutase [SOD]) levels. They were then divided into three groups $(n=6)$ and fed one of the following diets for 6 weeks:

Group I: Normal rabbit chow with high fat diet (HFD) $(5 \mathrm{ml} / \mathrm{kg})$.

Group II: Normal rabbit chow with high fat $(5 \mathrm{ml} / \mathrm{kg})$ and lycopene $(10 \mathrm{mg} / \mathrm{kg})$ orally.

Group III: Normal rabbit chow with HFD ( $5 \mathrm{ml} / \mathrm{kg})$ and atorvastatin $(5 \mathrm{mg} / \mathrm{kg})$ orally.

HFD: Mixture of coconut oil (from Marico Industries, Mumbai) and vanaspati ghee (from Hindustan Lever Ltd., Mumbai).

Lycopene powder: Purchased from Zenith Nutrition (Bangalore). Quality analysis was done from Bio-gen Extracts Pvt. Ltd. Bangalore.

Atorvastatin calcium powder: Procured as a gift sample from Emcure Pharmaceuticals (Pune).

\section{Method of preparation of HFD}

Edible coconut oil and vanaspati ghee mixed in the ratio of 2:3 respectively $\mathrm{v} / \mathrm{v} .{ }^{10}$

\section{Method of inducing hyperlipidaemia}

Above mentioned HFD, at a dose of $5 \mathrm{ml} / \mathrm{kg}$ body weight was fed to all the animals orally, along with normal rabbit chow for 6 weeks.

\section{Administration of lycopene}

Lycopene $10 \mathrm{mg} / \mathrm{kg}$, given orally to the second group by using the feeding tube along with the above diet for 6 weeks.

\section{Administration of atorvastatin}

Atorvastatin suspended in water containing $0.5 \%$ carboxymethylcellulose, ${ }^{11}$ administered orally to the third group with a feeding tube in the dose of $5 \mathrm{mg} / \mathrm{kg}$ along with the above diet for 6 weeks.

Fasting blood glucose was estimated by Optium Xceed glucometer. The levels of serum TC, TG, HDL and LDL were estimated to study the hypolipidemic activity of lycopene. 
Estimation of plasma SOD level was done to study the antioxidant effect of lycopene. All the above analyses were done before and after 6 weeks in both groups.

\section{Blood glucose}

Estimated by putting a drop of blood on the test strip inserted into the glucometer.

\section{Principle}

Glucose in the sample reacts with glucose oxidase and potassium ferricyanide. Electrons are generated producing a current, which is proportional to the glucose in the sample. After a specific reaction time, the glucose concentration in the sample is displayed.

Lipid profile: TC, TG and HDL were estimated manually on spectrophotometer. All reagents were purchased from Erba Manheim.

\section{Total cholesterol}

Measured by CHOD/PAP method (Erba Cholesterol DES assay kit) which depends on the oxidation of cholesterol by cholesterol oxidase to 7-hydroxyl - cholesterol. The hydrogen peroxide liberated then reacts with phenol and 4-amino antipyrine in the presence of peroxidase to yield a quinoneimine chromophore that is measured at $505 \mathrm{~nm}$.

\section{$T G S$}

Measured by GPO - Trinder method (Erba TGs DES assay kit) in which TGs undergo hydrolysis in the presence of lipoprotein lipase to form glycerol and free fatty acids. Glycerol reacts with adenosine triphosphate to form glycerol -3-phosphate which is oxidized to dihydroxyacetone phosphate. The hydrogen peroxide liberated then reacts with phenol and 4-amino antipyrine and 3,5-dichloro-hydroxybenzene sulfonate to yield a quinoneimine chromophore that is measured at $505 \mathrm{~nm}$.

\section{$H D L$}

Plasma HDL-C concentration (using Erba Liquixx HDL-C kit) was defined as the fraction of total cholesterol that remained in solution after precipitation of LDL and very LDL-C with magnesium chloride/phosphotungstic acid reagent added to the plasma sample. The plasma sample was centrifuged, and the clear supernatant assayed by the method used for estimation of plasma total cholesterol concentration.

\section{$L D L$}

Estimated by the Friedewald's equation. ${ }^{12}$ $\mathrm{LDL}=$ Total cholesterol-TG/5-HDL.
SOD: Measured by the method of Marklund and Marklund ${ }^{13}$

\begin{tabular}{|lcc|}
\hline Reagent & Control & Test \\
\hline Tris buffer & $3.0 \mathrm{ml}$ & $2.95 \mathrm{ml}$ \\
\hline Pyrogallol & $0.3 \mathrm{ml}$ & $0.3 \mathrm{ml}$ \\
\hline Serum & - & $0.05 \mathrm{ml}$ \\
\hline
\end{tabular}

Mix and measure the absorbance continuously for 4 mins at $420 \mathrm{~nm}$ at $30 \mathrm{sec}$ interval.

Calculation:

Absorbance reading to be taken for calculation is the reading at 3.5 mins minus reading at 0.5 mins. If absorbance reading of control is A and the test is B then:

$$
\mathrm{SOD}=\frac{\mathrm{A}-\mathrm{B}}{\mathrm{A} \times 50} \times \frac{100}{0.05} \text { units } / \mathrm{ml}
$$

\section{Statistical methods}

Statistical analysis was performed using statistical package SAS (Version 9.2 for Windows). Student's t-test (paired t-test) was used to compare the measurements taken at baseline and after 6 weeks within each group.

Results are expressed as mean \pm SEM and statistical significance between means was analyzed using one-way analysis of variance (ANOVA), followed by Tukey multiple comparison tests. $\mathrm{p}<0.05$ was considered to be statistically significant.

\section{RESULTS}

Table 1 shows the effect of HFD on the lipid profile, fasting blood glucose and SOD. The HFD resulted in a significant increase in blood lipid levels (TC, LDL-C and TG) and a significant decrease in the level of HDL-C and SOD. No significant difference was found in fasting blood glucose after HFD.

Table 2 shows the effect of additional supplementation of lycopene and atorvastatin in HFD group.

Figures 1 and 2 show the comparison between the parameters between HFD + lycopene and HFD + atorvastatin at the end of 6 weeks using one-way ANOVA.

Figure 1 shows a statistically significant $(\mathrm{p}<0.05)$ decrease in the level of TC, LDL-C and TG and increase in HDL-C levels in atorvastatin group when compared to lycopene group. However, there was no significant difference in blood glucose levels between the two groups.

Figure 2 shows statistically significant $(\mathrm{p}<0.05)$ increase in SOD level in the lycopene group when compared with atorvastatin group. 
Table 1: Effect of HFD on the lipid profile, fasting blood glucose and SOD levels.

\begin{tabular}{|lccc|}
\hline \multirow{2}{*}{ Parameter } & \multicolumn{2}{c}{ Mean \pm SEM } & p value \\
& Baseline & HFD & \\
\hline BSL $(\mathrm{mg} / \mathrm{dL})$ & $100.5 \pm 1.76$ & $103.5 \pm 1.56$ & 0.232 \\
\hline TC $(\mathrm{mg} / \mathrm{dL})$ & $106.6 \pm 2.06$ & $219.9 \pm 7.38$ & $<0.0001^{*}$ \\
\hline HDL $(\mathrm{mg} / \mathrm{dL})$ & $36.73 \pm 1.76$ & $25.01 \pm 1.17$ & $<0.0001^{*}$ \\
\hline TG $(\mathrm{mg} / \mathrm{dL})$ & $93.97 \pm 1.41$ & $149.5 \pm 4.11$ & $<0.0001^{*}$ \\
\hline LDL $(\mathrm{mg} / \mathrm{dL})$ & $51.09 \pm 0.39$ & $164.9 \pm 5.47$ & $<0.0001^{*}$ \\
\hline SOD (units) & $3.233 \pm 0.054$ & $2.292 \pm 0.050$ & $<0.0001^{*}$ \\
\hline
\end{tabular}

*Significant findings, BSL: Blood sugar level, TC: Total cholesterol, TG: Triglyceride, HDL: High-density lipoprotein, LDL: Low-density lipoprotein, SOD: Superoxide dismutase, HFD: High fat diet, SEM: Standard error of mean

Table 2: The effect of additional supplementation of lycopene and atorvastatin in HFD group on the lipid profile, fasting blood glucose and SOD levels.

\begin{tabular}{|lccc|}
\hline Parameter & \multicolumn{3}{c|}{$\begin{array}{c}\text { Mean } \pm \text { SEM } \\
\text { (HFD+ }\end{array}$} \\
\hline lycopene) & $\begin{array}{c}\text { (HFD+ } \\
\text { atorvastatin) }\end{array}$ \\
\hline BSL (mg/dL) & $103.5 \pm 1.56$ & $100.5 \pm 1.61$ & $99 \pm 1.12$ \\
\hline TC (mg/dL) & $219.9 \pm 7.38$ & $161.4 \pm 2.10 *$ & $146.5 \pm 2.27 * *$ \\
\hline HDL (mg/dL) & $25.01 \pm 1.17$ & $29.00 \pm 1.13 *$ & $31.29 \pm 1.28 * *$ \\
\hline TG (mg/dL) & $149.5 \pm 4.11$ & $123.6 \pm 2.87 *$ & $108.1 \pm 2.59 * *$ \\
\hline LDL (mg/dL) & $164.9 \pm 5.47$ & $106.9 \pm 0.77 *$ & $93.63 \pm 0.58 * *$ \\
\hline SOD (units) & $2.292 \pm 0.05$ & $2.987 \pm 0.03 *$ & $2.71 \pm 0.03 * *$ \\
\hline
\end{tabular}

*Significant findings $(\mathrm{p}<0.05)$ between HFD group and HFD+lycopene group. ${ }^{*}$ Significant findings $(\mathrm{p}<0.05)$ between HFD+lycopene group and HFD+atorvastatin group, BSL: Blood sugar level, TC: Total cholesterol, TG: Triglyceride, HDL: High-density lipoprotein, LDL: Low-density lipoprotein, SOD: Superoxide dismutase, HFD: High fat diet, SEM: Standard error of mean

\section{DISCUSSION}

Tomato is one of the most widely consumed fruits/ vegetable worldwide. It has been estimated that, in America, the average annual consumption of fresh tomatoes is approximately $8 \mathrm{~kg} /$ person and that of processed tomato products is $31 \mathrm{~kg} /$ person. ${ }^{14}$

Epidemiological studies indicate that consumption of tomatoes and tomato products is inversely associated with the prevalence of CVD. Tomatoes contain a plethora of ingredients. During recent years, the carotenoid lycopene has attracted much attention for its potentially beneficial cardiovascular effects. It is located mainly in tomato peels and contributes to the red color of tomatoes. A number of underlying mechanisms for the protective cardiovascular actions of lycopene have been suggested in cell culture studies. These include the inhibition of smooth muscle cell proliferation and foam cell formation, prevention

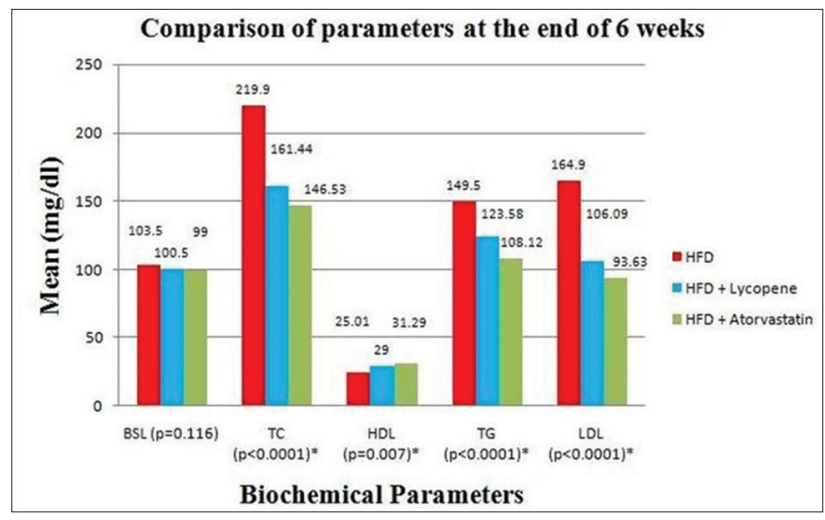

Figure 1: Comparison of parameters between high fat diet (HFD) + lycopene and HFD + atorvastatin at the end of 6 weeks. *Indicates significance between HFD + lycopene group and HFD + atorvastatin group.

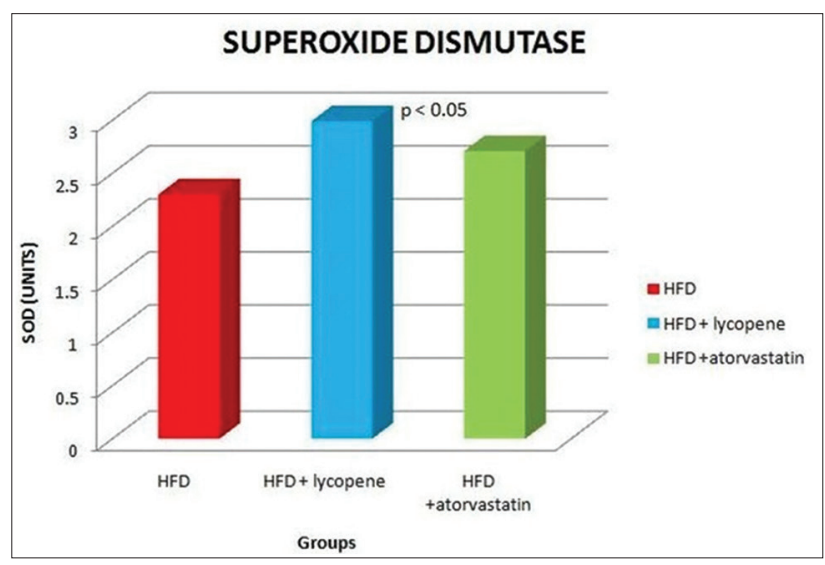

Figure 2: Comparison of level of superoxide dismutase between high fat diet (HFD) + lycopene and HFD + atorvastatin at 6 weeks. $p<0.05$ indicates statistical significance between HFD + lycopene and HFD + atorvastatin group.

of endothelial cell injury, modulation of cholesterol metabolism and inhibition of LDL oxidation and decrease of pro-inflammatory cytokines. ${ }^{15}$

In our study, we found the beneficial effects of pure lycopene powder in hyperlipidemia rabbits. There was a significant decrease in serum TC, LDL-C and total TG and a significant increase in serum HDL-C. This suggests that lycopene may play a role in the improvement of serum lipid profile and may possibly enhance LDL-C degradation. What are the mechanisms behind the cholesterol lowering effects of lycopene? Increased fecal cholesterol excretion, together with reduced liver $\mathrm{HMG}-\mathrm{CoA}$ reductase activity was shown after dietary lycopene intake in rabbits suggesting decreased intestinal cholesterol absorption and biosynthesis. ${ }^{1}$ However, the lipid lowering activity of $5 \mathrm{mg} / \mathrm{kg}$ atorvastatin was significantly better than that of lycopene. There was a significant difference in the levels of total cholesterol, total TGs, LDL and HDL when compared with lycopene group (Figure 1). 
Table 2 and Figure 2 show a significant increase in SOD level. This finding is common with the previous study. ${ }^{16}$ SOD is arguably the most crucial antioxidant in the body as it is responsible for disarming the most dangerous free radicals of all, the highly reactive superoxide. It reduces the radical superoxide $\left(\mathrm{O}_{2}^{-}\right)$to form hydrogen peroxide and oxygen $\left(\mathrm{H}_{2} \mathrm{O}_{2}\right.$ and $\mathrm{O}_{2}^{-}$). Although $\mathrm{H}_{2} \mathrm{O}_{2}$ is also a pro-oxidant compound, it is subsequently converted by enzyme catalase and glutathione peroxidase to simple water and oxygen. Hence, by strengthening the body's primary antioxidant system, this novel SOD boosting supplement may offer the powerful free radical protection and may play a protective role in reducing the oxidative stress that is implicated in atherosclerosis and other life threatening diseases. The antioxidant effect of lycopene was better than that of atorvastatin. Figure 1 shows no significant difference in fasting BSLs in both the groups.

\section{CONCLUSION}

These findings suggest that lycopene may have considerable therapeutic benefit as an antioxidant and hypolipidemic agent, but may not be effective as a hypoglycemic agent.

\section{ACKNOWLEDGMENTS}

The authors are thankful to M/s. Emcure Industries Ltd., Pune for supplying the gift sample of atorvastatin.

Funding: No funding sources Conflict of interest: None declared

Ethical approval: The study was approved by the Institutional Animal Ethics Committee

\section{REFERENCES}

1. Verghese M, Richardson JE, Boateng J, Shackelford LA, Howard C, Walker LT, et al. Dietary lycopene has a protective effect on cardiovascular disease in New Zealand male rabbits. J Biol Sci. 2008;8(2):268-77.

2. Riccioni G, Mancini B, Di Ilio E, Bucciarelli T, D’Orazio N. Protective effect of lycopene in cardiovascular disease. Eur Rev Med Pharmacol Sci. 2008;12(3):183-90.

3. $\mathrm{Hu}$ MY, Li YL, Jiang CH, Liu ZQ, Qu SL, Huang YM. Comparison of lycopene and fluvastatin effects on atherosclerosis induced by a high-fat diet in rabbits. Nutrition. 2008;24(10):1030-8.

4. Kamsiah J, Nafeeza M. Effect of nicardipine on fasting plasma lipids and apolipoproteins in male New Zealand white rabbits. Malays J Med Sci. 1999;6(2):5-11.

5. Rao AV, Rao LG. Carotenoids and human health. Pharmacol Res. 2007;55(3):207-16.

6. Mangels AR, Holden JM, Beecher GR, Forman MR, Lanza E. Carotenoid content of fruits and vegetables: an evaluation of analytic data. J Am Diet Assoc. 1993;93(3):284-96.

7. Britton G. Structure and properties of carotenoids in relation to function. FASEB J. 1995;9:1551-8.

8. Stahl W, Sies H. Lycopene: a biologically important carotenoid for humans? Arch Biochem Biophys. 1996;336(1):1-9.

9. Rashid S, Uffelman KD, Barrett PH, Lewis GF. Effect of atorvastatin on high-density lipoprotein apolipoprotein A-I production and clearance in the New Zealand white rabbit. Circulation. 2002;106(23):2955-60.

10. Shyamala MP, Venukumar MR, Latha MS. Antioxidant potential of Synzygium aromaticum (Gaertn.) Linn (Cloves) in rats fed with high fat diet. Indian J Pharmacol. 2003;35:99-103.

11. Suzuki M, Kakuta H, Takahashi A, Shimano H, Tada-Iida K, Yokoo T, et al. Effects of atorvastatin on glucose metabolism and insulin resistance in KK/Ay mice. J Atheroscler Thromb. 2005;12(2):77-84.

12. Friedewald WT, Levy RI, Fredrickson DS. Estimation of the concentration of low-density lipoprotein cholesterol in plasma, without use of the preparative ultracentrifuge. Clin Chem. 1972;18(6):499-502.

13. Marklund S, Marklund G. Involvement of the superoxide anion radical in the autoxidation of pyrogallol and a convenient assay for superoxide dismutase. Eur J Biochem. 1974;47(3):469-74.

14. Silaste ML, Alfthan G, Aro A, Kesäniemi YA, Hörkkö S. Tomato juice decreases LDL cholesterol levels and increases LDL resistance to oxidation. Br J Nutr. 2007;98(6):1251-8.

15. Lorenz M, Fechner M, Kalkowski J, Fröhlich K, Trautmann A, Böhm V, et al. Effects of lycopene on the initial state of atherosclerosis in New Zealand White (NZW) rabbits. PLoS One. 2012;7(1):e30808.

16. Subhash K, Bose C, Agrawal BK. Effect of short term supplementation of tomatoes on antioxidant enzymes and lipid peroxidation in type-II diabetes. Indian J Clin Biochem. 2007;22(1):95-8

doi: $10.5455 / 2319-2003 . i j b c p 20150229$

Cite this article as: Mulkalwar S, Rane B, Munjal

N, Golande P, Behera L. To evaluate and compare the hypoglycaemic, antioxidant and hypolipidaemic effect of lycopene with atorvastatin in hyperlipidaemic New Zealand white rabbits. Int J Basic Clin Pharmacol 2015;4:148-52 